\title{
Family-based association study of ITGB3 in autism spectrum disorder and its endophenotypes
}

\author{
Valerio Napolioni ${ }^{1,2}$, Federica Lombardi ${ }^{1,2}$, Roberto Sacco ${ }^{1,2}$, Paolo Curatolo ${ }^{3}$, Barbara Manzi ${ }^{3}$, \\ Riccardo Alessandrelli ${ }^{3}$, Roberto Militerni ${ }^{4}$, Carmela Bravaccio ${ }^{5}$, Carlo Lenti ${ }^{6}$, Monica Saccani ${ }^{6}$, \\ Cindy Schneider ${ }^{7}$, Raun Melmed ${ }^{8}$, Tiziana Pascucci ${ }^{9}, 10$, Stefano Puglisi-Allegra9 ${ }^{9,10}$, Karl-Ludvig Reichelt ${ }^{11}$, \\ Francis Rousseau ${ }^{12}$, Patricia Lewin ${ }^{12}$ and Antonio M Persico ${ }^{\star, 1,2}$
}

The integrin- $\beta 3$ gene (ITGB3), located on human chromosome 17q21.3, was previously identified as a quantitative trait locus (QTL) for 5-HT blood levels and has been implicated as a candidate gene for autism spectrum disorder (ASD). We performed a family-based association study in 281 simplex and 12 multiplex Caucasian families. ITGB3 haplotypes are significantly associated with autism (HBAT, global $\boldsymbol{P}=\mathbf{0 . 0 3 8}$ ). Haplotype H3 is largely over-transmitted to the affected offspring and doubles the risk of an ASD diagnosis (HBAT $P=0.005$; odds ratio $(O R)=2.000$ ), at the expense of haplotype $H 1$, which is undertransmitted (HBAT $P=0.018 ; O R=0.725$ ). These two common haplotypes differ only at rs 12603582 located in intron 11 , which reaches a $P$-value of 0.072 in single-marker FBAT analyses. Interestingly, rs 12603582 is strongly associated with pre-term delivery in our ASD patients $(P=0.008)$. On the other hand, it is SNP rs2317385, located at the $5^{\prime}$ end of the gene, that significantly affects 5 -HT blood levels (Mann-Whitney $U$-test, $P=0.001$; multiple regression analysis, $P=0.010$ ). No gene-gene interaction between ITGB3 and SLC6A4 has been detected. In conclusion, we identify a significant association between a common ITGB3 haplotype and ASD. Distinct markers, located toward the $5^{\prime}$ and $3^{\prime}$ ends of the gene, seemingly modulate 5-HT blood levels and autism liability, respectively. Our results also raise interest into ITGB3 influences on feto-maternal immune interactions in autism.

European Journal of Human Genetics (2011) 19, 353-359; doi:10.1038/ejhg.2010.180; published online 24 November 2010

Keywords: autism; integrin- $\beta$ 3; quantitative trait locus; SLC6A4; serotonin; serotonin transporter

\section{INTRODUCTION}

Autism spectrum disorder (ASD) is a complex neurodevelopmental disorder, characterized by different levels of impairment in social interaction and communication, by stereotypies and rigid patterns of behavior, and disease onset before 3 years of age (OMIM 209850). ${ }^{1}$ ASD is believed to primarily stem from genetic factors, based on the observation of $60-92 \%$ concordance rates in monozygotic twins vs $0-10 \%$ in dizygotic twins, with heritability estimated at or above $90 \% .^{2,3}$ The cause underlying autism in the majority of patients remains unknown, although several known medical conditions account for $\sim 10 \%$ of cases. ${ }^{4}$ ASD, similar to many other complex human disorders, does not show a simple inheritance pattern, as it may involve multiple common variants, each conveying a modest effect in epistatic interaction, rare variants with high penetrance, or perhaps more likely the coincidence of a rare variant acting upon a genetic background rendered vulnerable by a set of common variants. $^{2-5}$ Accordingly, familial aggregation of 'endophenotypes', heritable quantitative traits distributed continuously among ASD patients and first-degree relatives, can promote the search of genetic susceptibility factors in ASD.
Elevated whole-blood serotonin (5-HT) levels, one of the most consistent biological endophenotypes in autism research, is recorded in about one-third of cases. ${ }^{6}$ Autism-associated hyperserotonemia is indeed familial, ${ }^{7-9}$ and could either have a role in the etiological processes leading to the disease, or it could at least characterize a relatively homogeneous subgroup of ASD patients. Genes encoding proteins involved in 5-HT metabolism and neurotransmission include the integrin- $\beta 3$ subunit gene (ITGB3), located on human chromosome $17 \mathrm{q} 21.32$, which was identified as a quantitative trait locus (QTL) for 5-HT blood levels in the Hutterites. ${ }^{10,11}$ Interestingly, ITGB3 maps under a replicated linkage peak for autism. ${ }^{12,13}$ Furthermore, ITGB3 alleles have been found at least nominally associated with autism in all five studies performed to date, ${ }^{14-18}$ either alone or in interaction with allelic variants at the 5-HT transporter gene (SLC6A4). Several lines of evidence support functional interactions between ITGB3 and SLC6A4, which also affects 5-HT blood levels and is located on human chromosome $17 \mathrm{q} 11.1-\mathrm{q} 12 .{ }^{11}$ First, ITGB3 and SLC6A4 gene expression levels are correlated in human and mouse tissues. ${ }^{14}$ In fact, Slc6a4 mRNA levels map to the Itgb3 locus using QTL analysis in mouse hematopoietic stem cells, and non-coding

\footnotetext{
${ }^{1}$ Laboratory of Molecular Psychiatry and Neurogenetics, University ‘Campus Bio-Medico', Rome, Italy; 'Laboratory of Molecular Psychiatry and Psychiatric Genetics, Department of Experimental Neurosciences, IRCCS 'Fondazione Santa Lucia', Rome, Italy; ${ }^{3}$ Department of Child Neuropsychiatry, University 'Tor Vergata', Rome, Italy; ${ }^{4}$ Department of Child Neuropsychiatry, II University of Naples, Naples, Italy; ${ }^{5}$ Department of Pediatrics, University 'Federico II', Naples, Italy; ${ }^{6}$ Department of Child Neuropsychiatry, University of Milan, Milan, Italy; ${ }^{7}$ Center for Autism Research and Education, Phoenix, AZ, USA; ${ }^{8}$ Southwest Autism Research and Resource Center, Phoenix, AZ, USA; ${ }^{9}$ Department of Psychology and Centro 'Daniel Bovet', University 'La Sapienza', Rome, Italy; ${ }^{10}$ Laboratory of Behavioral Neurobiology, Department of Experimental Neurosciences, IRCCS 'Fondazione Santa Lucia', Rome, Italy; ${ }^{11}$ Department of Pediatric Research, Rikshospitalet, University of Oslo, Oslo, Norway; ${ }^{12}$ IntegraGen SA Genopole, Evry, France

*Correspondence: Dr AM Persico, Laboratory of Molecular Psychiatry and Neurogenetics, University 'Campus Bio-Medico', Via Alvaro del Portillo 21, Rome I-00128, Italy. Tel: +39 06 225419155; Fax: +39 06 501703333; E-mail: a.persico@unicampus.it

Received 22 April 2010; revised 17 September 2010; accepted 12 October 2010; published online 24 November 2010
} 
human polymorphisms in ITGB3 are associated with both ITGB3 and SLC6A4 expression levels. ${ }^{14}$ Second, the integrin receptor composed of an $\alpha \mathrm{IIb}$ subunit and of the $\beta 3$ subunit encoded by the ITGB3 gene, was recently identified as a novel component of the SLC6A4 regulatory protein complex. ${ }^{14}$ Also the Leu33Pro ITGB3 SNP (rs5918) modulates SLC6A4 trafficking and transport activity. ${ }^{19}$ Finally, several recently published studies have described significant SLC6A4 and ITGB3 interactions for both autism risk and 5-HT blood levels, with a male-specific effect. ${ }^{10,14-17,20}$

Despite these positive findings, several inconsistencies complicate their interpretation, possibly because of clinical and genetic heterogeneity in ASD. In particular, different alleles seem to be associated with autism and/or serotoninemia in independent samples at the ITGB3 and SLC6A4 loci. ${ }^{16,18}$ Linkage disequilibrium (LD) blocks associated with autism and/or serotoninemia are not consistent, with different studies pointing toward either the $5^{\prime}$ or the $3^{\prime}$ ends of the ITGB3 locus. ${ }^{14-18,20}$ Conceivably, these inconsistencies could stem from different causative variants occurring on distinct marker haplotype backgrounds.

This study was thus undertaken (a) to replicate and extend previous findings, by fine mapping the association between ITGB3 and ASD using a family-based approach; (b) to determine the effect of ITGB3 alleles on biochemical and morphological quantitative endophenotypes, including 5-HT blood levels; (c) to test for gene-gene interactions between ITGB3 and SLC6A4 in reference to autism risk and 5-HT blood levels, (d) to correlate ITGB3 and SLC6A4 genotypes with clinical features, as well as with patient and family history variables.

\section{MATERIALS AND METHODS}

\section{Subjects}

A total of 281 simplex and 12 multiplex families with a non-syndromic autistic proband were recruited for this study, including 306 ASD patients, 106 unaffected siblings, and 577 parents; total genotyped $N=989$. Demographic and clinical characteristics of our clinical sample, as well as endophenotypic measures for head circumference, serotonin (5-HT) blood levels and global peptiduria, are summarized in Table 1 . The composition by recruiting site is presented in Supplementary Table S1. Diagnostic screening procedures used to exclude syndromic autism have been previously described. ${ }^{21}$ Briefly, patients fulfilling DSM-IV diagnostic criteria for autistic disorder ${ }^{1}$ were screened for non-syndromic autism using MRI, EEG, audiometry, urinary amino acid and organic acid measurements, cytogenetic and fragile-X testing. Patients with dysmorphic features were excluded even in the absence of detectable cytogenetic alterations. Patients with sporadic seizures (ie, $<1$ every 6 months) were included; patients with frequent seizures or focal neurological deficits were excluded. The M:F ratio in ASD patients is 7.3:1. Autistic behaviors were assessed using the official Italian version of the Autism Diagnostic Observation Schedule ${ }^{22}$ and the Autism Diagnostic Interview-Revised; ${ }^{23}$ adaptive functioning was assessed using the Vineland Adaptive Behavior Scales; IQ was determined using either the Griffith Mental Developmental Scales, the Colored Raven Matrices, the Bayley Developmental Scales or the Leiter International Performance Scale. ${ }^{21}$ All parents gave written informed consent for themselves and for their children, using the consent form approved by the IRB of UCBM (Rome, Italy).

\section{Genotyping}

Genomic DNA was extracted from whole blood ${ }^{24}$ and quantified in triplicate by PicoGreen (Molecular Probes, OR, USA). On the basis of HapMap phase II (release 21) CEU population data, four independent LD blocks were identified within ITGB3 (chromosome 17: 42684-42750 kb) using the 'solid spine of LD' algorithm with a minimum $D$-value of 0.8 . In all, 10 tagging SNPs were selected using Tagger from Haploview v4.2 $2^{25}\left(r^{2}>0.75\right.$ and minor allele frequency $>0.05$, aggressive tagging, LOD threshold for multimarker test $=3$ ). All SNPs previously associated with autism were comprised by applying the 'force-include'
Table 1 Demographic, clinical, and endophenotypic characteristics of the autistic sample

\begin{tabular}{|c|c|c|c|}
\hline & $\mathrm{N}$ & Mean/median & Range \\
\hline Age in years (mean \pm SEM): & $N=306$ & $9.18 \pm 0.33$ & $2-33$ \\
\hline Median VABS scores: & $N=137$ & & \\
\hline Communication & & 69.0 & $19-128$ \\
\hline Daily living skills & & 67.0 & $14-170$ \\
\hline Socialization & & 66.0 & $25-140$ \\
\hline Motor skills & & 80.0 & $25-128$ \\
\hline Composite & & 60.0 & $19-137$ \\
\hline $\begin{array}{l}\text { Head circumference: } \\
\text { (median percentile } \pm I Q R / 2 \text { ) }\end{array}$ & $N=265$ & $82.5 \pm 23.75$ & $2.5-98.5$ \\
\hline $\begin{array}{l}\text { Serotonin blood levels: } \\
\text { (mean } \mathrm{ng} / \mathrm{ml} \pm \text { SEM) }\end{array}$ & $N=158$ & $329.5 \pm 20.9$ & $31.0-987$. \\
\hline
\end{tabular}

Urinary oligopeptiduria:

(mean $\mu \mathrm{m}^{2} \pm \mathrm{SEM}$ )

$N=231 \quad 346.2 \pm 16.5$

$57-1213$

N

Percent (\%)

\section{Gender:}

Male

Female

269

87.9

$\mathrm{M} / \mathrm{F}$ ratio

37

12.1

\section{Family type:}

Simplex

Multiplex

281

12

95.9

DSM-IV diagnosis:

Autistic disorder

Asperger syndrome

207

67.6

PDD - NOS

27

8.8

23.6

IQ $(N=71):$

$\begin{array}{lll}>70 & 18 & 25.4 \\ <70 & 53 & 74.6\end{array}$

$<70$

74.6

Abbreviations: IQR/2, semi-interquartilic range; PDD - NOS, pervasive developmental disorder not otherwise specified.

procedure of Haploview, in addition to rs11650072, which provides further coverage of the 3 '-flanking region (Supplementary Table S2). The ITGB3 genotyping was performed using the Applied Biosystems SNPlext Genotyping System (Applied Biosystems, CA, USA). All samples were electrophoretically separated on a 3730 DNA Genetic Analyzer (Applied Biosystems), and automated allele calls and genotype clustering of each individual sample was performed by Applied Biosystems GeneMapper Software (version 3.5). ITGB3 SNP rs5918 was genotyped using the TaqMan SNP genotyping assay (Applied Biosystems) on the ABI Prism 7900HT and analyzed with the SDS software (Applied Biosystems). SLC6A4 5-HTTLPR genotyping was performed as previously described. ${ }^{26}$

\section{Endophenotype measures}

Serotonin levels were measured in all family members from platelet-rich plasma, obtained by centrifuging whole blood within $20 \mathrm{~min}$ of venipuncture at $140 \mathrm{~g}$ for $25 \mathrm{~min}$ at $4^{\circ} \mathrm{C} ; 1 \mathrm{ml}$ of supernatant was stored at $-80^{\circ} \mathrm{C}$ and assessed by HPLC, as described. ${ }^{27}$ Urinary peptide excretion analysis was performed by HPLC in ASD patients and first-degree relatives using the first morning urine samples, as described. ${ }^{28}$ The total area of peaks under the $215 \mathrm{~nm}$ absorption curve (AUC) in the peptide region following the hippuric acid peak was calculated and expressed in $\mu \mathrm{m}^{2}$. Head circumference was measured in ASD patients and unaffected siblings by trained physicians using a non-stretchable plastic measuring tape graded in millimeters, placed over the maximum frontal-occipital head perimeter. ${ }^{21}$ 


\section{Statistical analysis}

Mendelian inheritance was verified using Pedcheck. ${ }^{29}$ Hardy-Weinberg equilibrium (HWE) was tested using Haploview v4.2 (available at http:// www.broad.mit.edu/mpg/haploview/index.php) ${ }^{25}$ applying a Bonferroni's correction for multiple testing $(P<0.05 / 11$ SNPs yields $P<0.0045)$. LD analysis was performed using Haploview, and defining LD blocks based on the solid spine of LD algorithm. ${ }^{25}$ Differences in LD structure recorded applying the confidence interval $s^{30}$ and the four-gamete rule ${ }^{31}$ algorithms are also reported. Family-based single-marker and haplotype association tests were performed using FBAT (available at http://www.biostat.harvard.edu/ fbat/fbat.htm), under an additive model and applying option $-e$, as suggested for candidate genes under known linkage peaks. ${ }^{32}$ The HBAT procedure in FBAT was also used to estimate haplotype frequencies, to compute a global $P$-value, and to provide an 'exact' $P$-value using Monte Carlo tests (option $-p$ ) for the global test $\left(\chi^{2}\right.$ sum $P$ ), for each haplotype separately, and for the minimum observed $P$-value among all haplotypes (minimal $P) \cdot{ }^{32}$ Haplotype odds ratios (ORs) were determined using UNPHASED (available at http://www.mrc-bsu.cam.ac.uk/ personal/frank/software/unphased/). ${ }^{33}$ Quantitative traits were analyzed by quantitative transmission/disequilibrium test (qTDT), as implemented by the FBAT software ${ }^{32}$ and by parametric or non-parametric (Kruskal-Wallis) ANOVA, or by Mann-Whitney $U$-tests based on genotype distributions, applying a stringent Bonferroni's correction for multiple testing (4 markers $\times 3$ phenotypes, $P=0.5 / 12=0.0041)$. Gene-gene interaction analyses were performed with the two-locus transmission/disequilibrium test (TDT) method, ${ }^{34}$ which has been implemented as a Stata program 'pseudocc' (http://www-gene.cimr.cam. ac.uk/clayton/software/stata/). Data are expressed as mean \pm SEM, except for head circumference, which is expressed as median \pm semi-interquartilic range (IQR/2). Head circumference measures were transformed into percentiles using sex- and age-specific standard tables, as described. ${ }^{35}$ Two-tail $P$-values are reported. To correct for multiple comparisons in single-marker analyses, statistical significance was set at $P<0.0016$ : this threshold accounts for testing of eight effectively independent markers (seven on ITGB3 and one on SLC6A4), as determined using the Nyholt SNPSpD method, ${ }^{36}$ (available at http://genepi.qimr.edu.au/ general/daleN/SNPSpD/), and four phenotypes (autism, serotoninemia, peptiduria, and head circumference; Supplementary Methods). Nominal $P$-values obtained by Pearson's $\chi^{2}$-tests are reported for clinical, patient and family history variables, given the exploratory nature of these associations.

\section{RESULTS}

\section{ITGB3 haplotype analysis}

The 11 ITGB3 SNPs are in HWE both in the entire sample and analyzing separately mothers, fathers, autistic, and unaffected siblings, with the exception of rs3809863, which has been excluded from subsequent analyses (Supplementary Table S2). The results of LD analysis are displayed in Figure 1. All three algorithms applicable for LD block definition consistently identify at the $3^{\prime}$ end one LD block, encompassing the three SNPs located most downstream, and at the $5^{\prime}$ end rs2317385 (SNP1), which is not associated with any other SNP and is part of an independent LD block located upstream of ITGB3; in between SNP1 and the $3^{\prime}$ LD block, SNPs 2 to 7 span another LD block showing increasing size when defined according to the confidence intervals, four-gamete rule, or solid spine of LD algorithms, respectively (Figure 1). Mean $r^{2}$ is 0.15 , confirming a relatively low overall inter-SNP correlation, consistent with the selection of tagging SNPs for genotyping (Figure 1).

ITGB3 haplotypes show a statistically significant association with autism (HBAT global $P=0.038$; whole marker permutation tests yield sum $P=0.017$ and minimal $P=0.011$, after 100000 iterations). Haplotype $\mathrm{H} 3$ is transmitted from heterozygous parents to their autistic offspring significantly more often than expected by chance $(P=0.005)$, whereas haplotype $\mathrm{H} 1$ shows the opposite trend $(P=0.018$; Table 2). In terms of ORs, haplotype $\mathrm{H} 3$ doubles the risk of autism $\left(\mathrm{OR}=2.000 ; \chi^{2}=8.426 ; P=0.003\right)$, whereas haplotype $\mathrm{H} 1$ marginally reduces disease risk $\left(\mathrm{OR}=0.725 ; \chi^{2}=3.572 ; P=0.059\right)$.

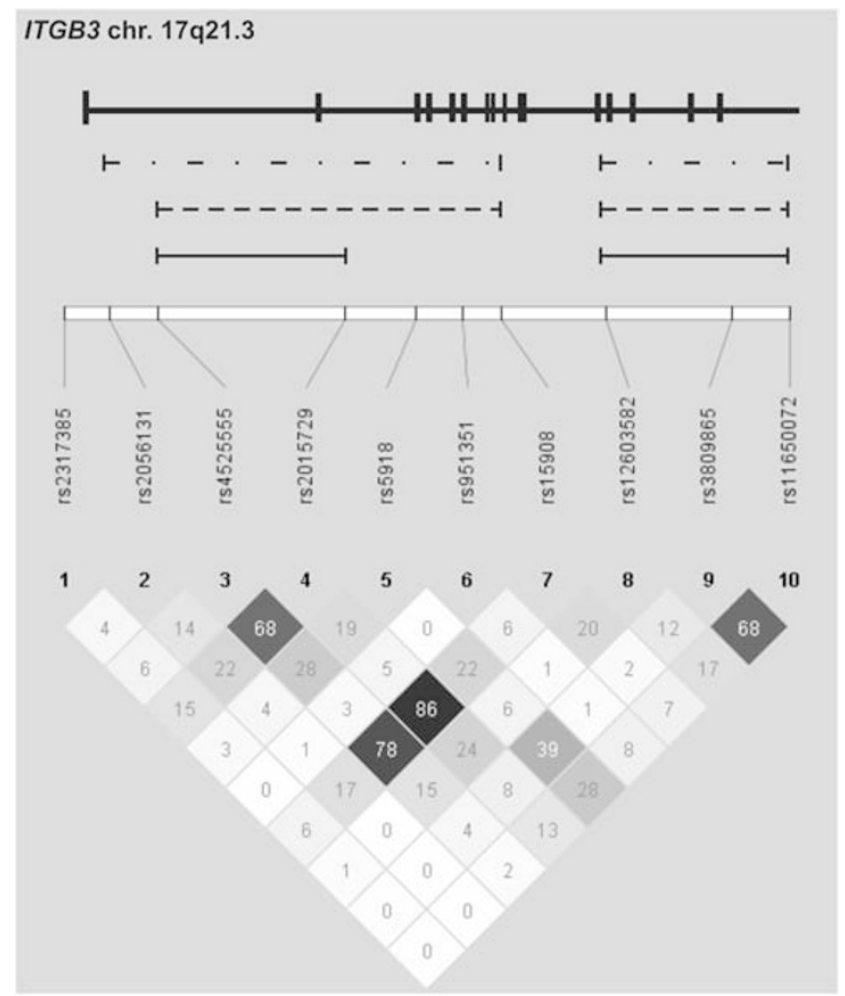

Figure 1 ITGB3 exon-intron structure, genotyped SNPs, and linkage disequilibrium expressed in $r^{2}$. Haplotype blocks defined according to the confidence interval, four-gamete rule, and solid spine of LD algorithms are shown above by solid, broken, and dotted/broken lines, respectively.

\section{Single-marker analyses}

Interestingly, haplotypes $\mathrm{H} 1$ and $\mathrm{H} 3$ differ only at SNP rs12603582 located in intron 11. Using single-marker FBAT and TDT analyses, rs 12603582 was the only marker showing a trend toward the preferential transmission of allele $\mathrm{G}$ in the overall sample (FBAT additive model, $P=0.072$; TDT, $P=0.057)$, in autistic males only $(N=236$, FBAT $P=0.049)$ and in simplex families $(N=281$, FBAT $P=0.053$; Supplementary Table S3). No significant evidence of protective alleles, preferentially transmitted from heterozygous parents to unaffected siblings, was found using both haplotype and single-marker analyses (data not shown).

\section{Gene-gene interaction between ITGB3 and SLC6A4 in autism}

SLC6A4 5-HTTLPR genotypes are in HWE and show no association with autism in this sample (TDT LRS $=0.562,1$ d.f., $P=0.454$; FBAT $P=0.432$ ). To test for interaction between ITGB3 and SLC6A4, we applied a two-locus TDT approach, ${ }^{34}$ crossing ITGB3 genotypes either at rs5918 (SNP5, Leu33Pro), rs12603582 (SNP8), or rs3809865 (SNP9), with SLC6A4 genotypes at the 5-HTTLPR. No evidence of epistatic effects on autism risk was detected in our entire sample, in males only, or in simplex families.

\section{Quantitative endophenotypes: single-gene effects and gene-gene interactions for ITGB3 and SLC6A4}

Single-marker qTDT analyses show a nominal association of ITGB3 SNP1, rs2317385, with 5-HT blood levels $(P=0.016)$ and peptiduria $(P=0.041)$, not reaching the significance threshold set by the Nyholt SNPSpD method to control for multiple testing $(P<0.0016)$. However, the association with 5-HT blood levels survives even a 
Table 2 ITGB3 haplotypes are associated with autism: (a) haplotype structure at the ITGB3 locus. (b) Haplotype family-based association tests performed using HBAT, under an additive model $(-e)^{32}$

$\left(a^{a}\right.$

\begin{tabular}{|c|c|c|c|c|c|c|c|c|c|c|c|}
\hline \multicolumn{12}{|c|}{ ITGB3 SNPS } \\
\hline & 1 & 2 & 3 & 4 & 5 & 6 & 7 & 8 & 9 & 10 & Estimated \\
\hline Haplotype & rs2317385 & rs2056131 & rs4525555 & rs2015729 & rs5918 & rs951351 & rs15908 & rs12603582 & rs3809865 & rs11650072 & Frequency \\
\hline $\mathrm{H} 2$ & G & G & $\mathrm{T}$ & A & C & G & A & G & $\mathrm{T}$ & $\mathrm{T}$ & 0.125 \\
\hline H3 & G & G & C & G & $T$ & G & C & G & A & C & 0.103 \\
\hline $\mathrm{H} 4$ & G & G & $\mathrm{T}$ & A & $\mathrm{T}$ & G & A & G & A & C & 0.102 \\
\hline $\mathrm{H} 7$ & G & A & C & G & $\mathrm{T}$ & G & C & G & $T$ & $\mathrm{~T}$ & 0.074 \\
\hline H8 & G & A & C & G & $\mathrm{T}$ & G & C & $\mathrm{T}$ & A & C & 0.058 \\
\hline H9 & G & G & C & A & $\mathrm{T}$ & A & A & G & A & $\mathrm{T}$ & 0.041 \\
\hline $\mathrm{H} 10$ & $A$ & G & C & A & $\mathrm{T}$ & G & C & G & $\mathrm{T}$ & $\mathrm{T}$ & 0.027 \\
\hline $\mathrm{H} 11$ & A & G & C & G & $\mathrm{T}$ & G & C & $\mathrm{T}$ & A & C & 0.011 \\
\hline H12 & G & A & C & G & $\mathrm{T}$ & G & C & G & A & $\mathrm{T}$ & 0.011 \\
\hline H17 & G & G & $\mathrm{T}$ & A & $\mathrm{T}$ & G & A & G & $\mathrm{T}$ & T & 0.005 \\
\hline $\mathrm{H} 18$ & $\mathrm{G}$ & G & C & G & $\mathrm{T}$ & G & C & G & $\mathrm{T}$ & $\mathrm{T}$ & 0.005 \\
\hline H19 & A & G & $\mathrm{T}$ & A & $\mathrm{T}$ & G & A & G & A & $\mathrm{T}$ & 0.005 \\
\hline
\end{tabular}

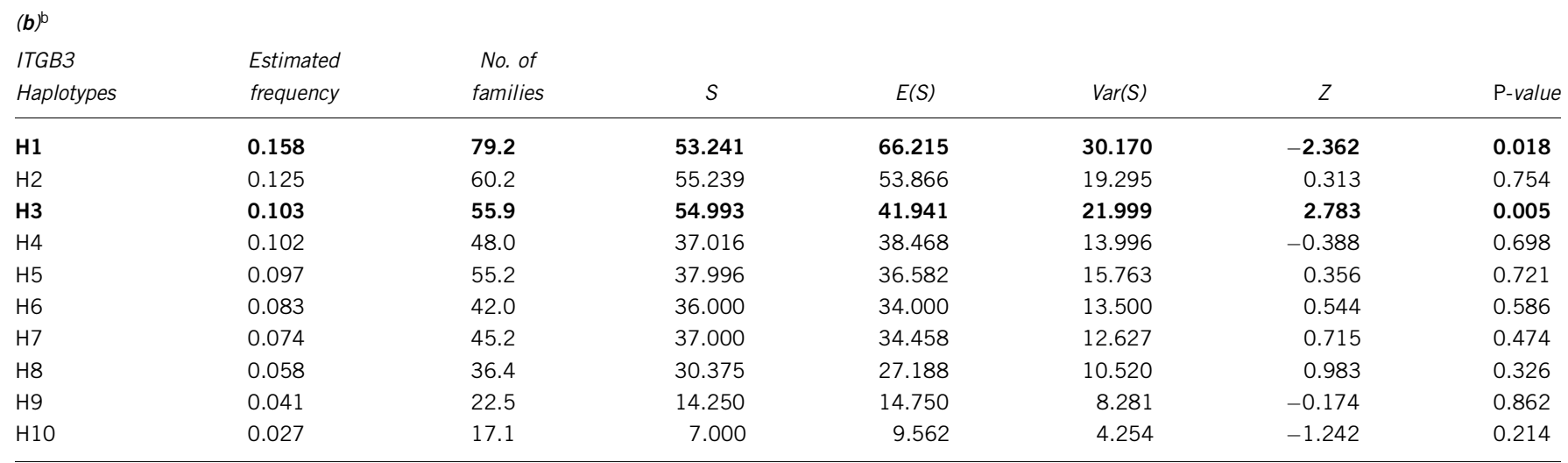

anly haplotypes with estimated frequencies $\geq 0.005$ are listed.

bHaplotype global $P$-value for HBAT is $P=0.038$; whole marker permutation tests yield $\gamma^{2}$ sum $P=0.017$ and minimal $P=0.011$, after 100000 iterations.

Haplotypes $\mathrm{H} 1$ and $\mathrm{H} 3$ highlighted in bold are significantly under- and over-transmitted, respectively, from heterozygous parents to the affected offspring.

stringent Bonferroni correction in quantitative analyses $(P=0.001$; Table 3). Multiple regression analysis reveals ITGB3 SNP1, rs2317385 as the only SNP significantly affecting 5-HT blood levels $(P=0.010)$, whereas SLC6A4 5-HTTLPR reaches marginal significance $(P=0.070)$, with no evidence of gene-gene interactions $(P=0.651$; Supplementary Figure 1). SLC6A4 5-HTTLPR provides negligible contributions to the percentage of variance in 5-HT blood levels attributable to ITGB3 rs 2317385 alone, which passes from 5.5 to $6.0 \%$.

Association of ITGB3 and SLC6A4 genotypes with clinical variables Allele T at rs12603582 (SNP8 in ITGB3) is strongly associated with a shorter pregnancy duration ending in pre-term delivery $\left(\chi^{2}=9.78\right.$, 2 d.f., $P=0.008$ ), whereas the Pro33 allele at rs5918 (SNP5) is nominally associated with obstetric complications in the mother $\left(\chi^{2}=6.40,2\right.$ d.f., $\left.P=0.041\right)$, allergies in the patient $\left(\chi^{2}=6.74,2\right.$ d.f., $P=0.034)$, and modulation of the pain threshold, as reported by parents $\left(\chi^{2}=6.98,2\right.$ d.f., $P=0.030$; Table 4$)$. On the other hand, SLC6A4 5-HTTLPR is nominally associated with several immunerelated clinical variables and with parent-reported elevated pain thresholds (Table 4). No association with any clinical variable was found for rs2317385 (SNP1).

\section{DISCUSSION}

This study reports a significant association between ASD and an ITGB3 allele marked here by haplotype $\mathrm{H} 3$, which doubles the risk of autism in our sample. The autism-associated haplotype is primarily defined by rs 12603582 , located toward the $3^{\prime}$ end of the gene, whereas 
Table 3 Head circumference, serotonin blood levels, and global peptiduria by ITGB3 and SLC6A4 genotypes

\begin{tabular}{|c|c|c|c|c|c|c|c|}
\hline GENOTYPES & & Head circumference & & 5-HT blood levels & & Global peptiduria & \\
\hline ITGB3, SNP1: & $\mathrm{G} / \mathrm{G}$ & $82.5 \pm 47.5 N=144$ & $K-W \chi^{2}=3.542$ & $317.3 \pm 25.0 N=99$ & Pairwise $U$-test: & $345.3 \pm 23.0 N=145$ & $\mathrm{~K}-\mathrm{W} \chi^{2}=1.676$ \\
\hline rs2317385 & $\begin{array}{l}G / A \\
A / A\end{array}$ & $\begin{array}{l}75.0 \pm 47.4 N=60 \\
97.5 N=3\end{array}$ & 2 d.f., $P=0.170$ & $\begin{array}{l}468.9 \pm 44.0 \quad N=36 \\
261.0 N=1\end{array}$ & $\begin{array}{l}\mathrm{GG} \text { vs } \mathrm{GA}+\mathrm{AA} \\
\mathrm{U}=1087.0 \\
P=0.001\end{array}$ & $\begin{array}{l}309.6 \pm 32.3 N=41 \\
267.0 \pm 168.0 N=3\end{array}$ & 2 d.f., $P=0.433$ \\
\hline ITGB3, SNP5: & $\mathrm{T} / \mathrm{T}$ & $82.5 \pm 47.5 N=182$ & $\mathrm{~K}-\mathrm{W} \chi^{2}=1.377$ & $313.7 \pm 23.9 N=102$ & $\mathrm{~K}-\mathrm{W} \chi^{2}=1.207$ & $344.3 \pm 17.4 N=151$ & $\mathrm{~K}-\mathrm{W} \chi^{2}=0.793$ \\
\hline rs5918 & $\begin{array}{l}\mathrm{C} / \mathrm{T} \\
\mathrm{C} / \mathrm{C}\end{array}$ & $\begin{array}{l}75.0 \pm 47.5 N=74 \\
82.5 \pm 47.5 N=9\end{array}$ & 2 d.f., $P=0.502$ & $\begin{array}{l}314.2 \pm 29.2 N=48 \\
421.1 \pm 104.5 N=8\end{array}$ & 2 d.f., $P=0.547$ & $\begin{array}{l}355.3 \pm 37.4 N=74 \\
281.2 \pm 59.0 N=6\end{array}$ & 2 d.f., $P=0.673$ \\
\hline $\begin{array}{l}\text { ITGB3, SNP8: } \\
\text { rs12603582 }\end{array}$ & $\begin{array}{l}\mathrm{G} / \mathrm{G} \\
\mathrm{G} / \mathrm{T} \\
\mathrm{T} / \mathrm{T}\end{array}$ & $\begin{array}{l}82.5 \pm 47.5 N=121 \\
78.7 \pm 47.5 N=74 \\
86.2 \pm 47.5 N=10\end{array}$ & $\begin{array}{l}K-W \chi^{2}=0.408 \\
2 \text { d.f., } P=0.815\end{array}$ & $\begin{array}{l}368.4 \pm 31.6 N=77 \\
353.7 \pm 35.0 N=52 \\
311.2 \pm 97.5 N=5\end{array}$ & $\begin{array}{l}\mathrm{K}-\mathrm{W} \chi^{2}=0.181 \\
2 \text { d.f., } P=0.913\end{array}$ & $\begin{array}{l}331.6 \pm 26.1 N=107 \\
340.6 \pm 25.8 N=74 \\
366.1 \pm 171.9 N=8\end{array}$ & $\begin{array}{l}\mathrm{K}-\mathrm{W} \chi^{2}=1.586 \\
2 \text { d.f., } \mathrm{P}=0.453\end{array}$ \\
\hline $\begin{array}{l}\text { SLC6A4, } \\
\text { 5-HTTLPR }\end{array}$ & $\begin{array}{l}S / S \\
S / L \\
L / L\end{array}$ & $\begin{array}{l}82.5 \pm 47.3 N=69 \\
82.5 \pm 47.5 N=123 \\
90.0 \pm 35.0 N=82\end{array}$ & $\begin{array}{l}K-W \chi^{2}=4.329 \\
2 \text { d.f., } P=0.115\end{array}$ & $\begin{array}{l}390.3 \pm 43.9 N=41 \\
282.0 \pm 21.4 N=80 \\
385.0 \pm 41.0 N=49\end{array}$ & $\begin{array}{l}\mathrm{K}-\mathrm{W} \chi^{2}=4.821 \\
2 \text { d.f., } P=0.09\end{array}$ & $\begin{array}{l}325.2 \pm 23.7 N=50 \\
364.4 \pm 28.4 N=114 \\
329.6 \pm 22.9 N=76\end{array}$ & $\begin{array}{l}\mathrm{K}-\mathrm{W} \chi^{2}=0.260 \\
2 \text { d.f., } P=0.878\end{array}$ \\
\hline
\end{tabular}

Abbreviation: $\mathrm{K}-\mathrm{W}$, Kruskal-Wallis test (non-parametric ANOVA).

Data are expressed as mean \pm SEM, except for head circumference, which is expressed as median \pm semi-interquartilic range (IQR/2). Nominal $P$-value are reported; highlighted in bold, statistically significant results surviving Bonferroni's correction (significance set at $P<0.0041$ ).

Table 4 Association of ITGB3 and SLC6A4 genotypes with clinical variables

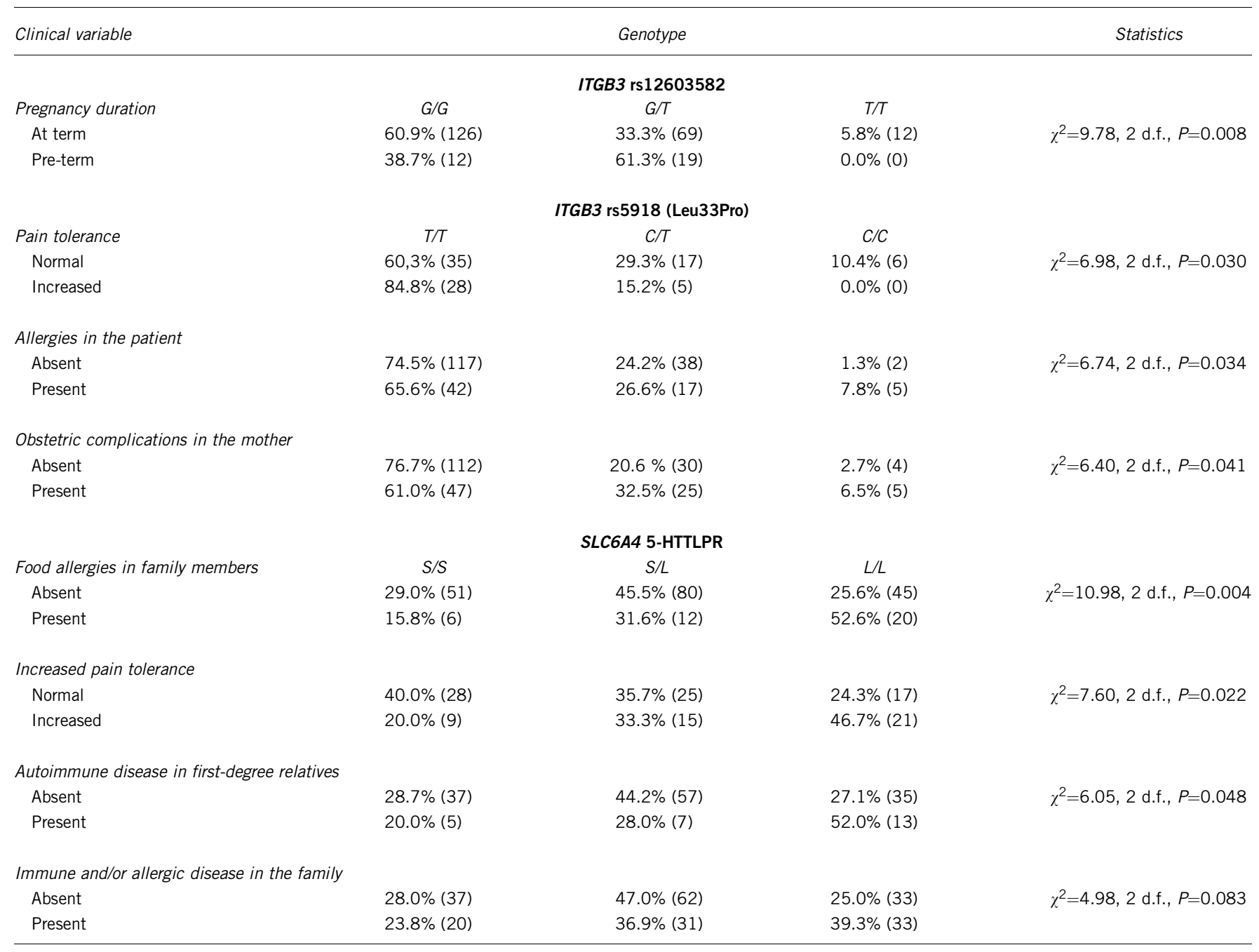


rs 2317385 , located at the $5^{\prime}$ end, is significantly associated with 5-HT blood levels. Conversely, the former SNP shows no association with serotoninemia, and the latter provides no contribution to autism risk. Hence, multiple functional ITGB3 polymorphisms located in different parts of the gene are seemingly responsible for contributions to autism liability and to 5-HT blood levels in our sample.

The existence of at least two distinct functional genetic variants at the ITGB3 locus is highly compatible with previous reports on autism and other disorders, such as asthma and allergies. ${ }^{37,38}$ At the $5^{\prime}$ end of the gene, rs2317385 is associated with higher 5-HT blood levels both in our sample and in a previously reported healthy population sample recruited in Chicago. ${ }^{16}$ This variant was never found associated with autism risk in earlier studies. ${ }^{14-18}$ Toward the $3^{\prime}$ end, we apparently fail to replicate the positive nominal association between autism and SNPs rs5918, rs15908, and rs3809865 located in exon 3, exon 9, and 3' UTR, respectively. However, at least for rs5918, the initial report of an association with $\mathrm{ASD}^{16}$ was not replicated in several follow-up studies. ${ }^{17,18}$ On the other hand, rs15908, and rs3809865 are all located at a short distance from our SNP rs12603582 (Figure 1). Conceivably, the association of a single putative functional variant with different markers in different samples, could be well explained by interethnic differences in LD pattern, in the presence of $r^{2}$ values as low as those shown in Figure 1. This discrepancy between $r^{2}$ and LD block definition based on $D$ value is because of the very different frequencies of associated alleles at these SNPs. The association of the major allele at each SNP with the minor allele at the other SNP, decreases dramatically the informativeness of major alleles at each SNP in reference to alleles present at the other SNP. ${ }^{39}$ Regardless, the existence of separate $5^{\prime}$ and $3^{\prime}$ functional variants contributing to serotoninemia and autism, respectively, remains a consistent observation, closely resembling the association patterns reported for asthma and wheezing $v s$ allergies and IgE levels, also associated with distinct $5^{\prime}$ and $3^{\prime}$ markers. ${ }^{37,38}$

In spite of the extraordinary challenge posed by the complex pathogenetic processes underlying ASD, different lines of evidence are starting to converge on some basic mechanisms. The prominent increase in pre-term births detected here among allele $\mathrm{T}$ carriers at SNP rs12603582, and the absence of T/T genotype carriers among the autistic offspring, strongly point toward a deleterious effect of the $\mathrm{T}$ allele during pregnancy, which would then translate into the preferential transmission of allele $G$ from heterozygous parents to autistic offspring. Additional contributions to the occurrence of obstetric complications and of repeated spontaneous abortions in mothers of autistic individuals come from the Pro33 allele at rs5918. Importantly, Pro33 is in LD with the G, and not with the T allele, at rs12603582, indicating that the two SNPs may be independently influencing early life liability. This is not entirely surprising, as neonatal alloimmune thrombocytopenia, the most common cause of severe thrombocytopenia in otherwise healthy term infants, is due to a feto-maternal mismatch for human platelet alloantigens encoded by the ITGB3 gene. ${ }^{40}$ Importantly, the enhanced risk for early fetal loss conferred by the Pro33 allele has been previously recorded in the general population, ${ }^{41}$ whereas to our knowledge no previous evidence of involvement for rs12603582 has been produced. Hence, the latter may function specifically in families carrying an autism-predisposing genetic background. Finally, contrary to previous studies, ${ }^{14,15,17,18}$ our sample provides no evidence of significant gene-gene interaction between SLC6A4 and ITGB3. Instead, the L/L genotype at SLC6A4 shows nominal associations with immunological conditions and increased pain tolerance, a result quite compatible with well-known 5-HT roles in adaptive immune responses and in determining the sensitive threshold to noxious stimuli. ${ }^{42,43}$
In conclusion, our results confirm and extend previous findings, supporting the existence of relevant influences by ITGB3 gene variants on autism liability and on 5-HT blood levels. We further describe a significant association between early fetal loss, preterm delivery, and obstetric complications in the mothers of autistic children, with ITGB3 gene variants active either in the general population, as previously reported, ${ }^{40,41,44}$ or possibly affecting the feto-maternal unit only in autism spectrum families. Collectively, the results of this and of previous studies spur strong interest into the identification and functional characterization of ITGB3 variants functionally implicated in the underpinnings of autism.

\section{CONFLICT OF INTEREST}

The authors declare no conflict of interest.

\section{ACKNOWLEDGEMENTS}

We acknowledge all the families who participated in this study, Roberto Rigardetto, Marina Gandione, Simona Trillo, and Maria Paola Santangelo for contributing to patient recruitment/data collection, and Jerome Carayol for critical reviewing of manuscript. This work was supported by the Italian Ministry for University, Scientific Research and Technology (PRIN n.2006058195), the Italian Ministry of Health (RFPS-2007-5-640174), the Autism Speaks Foundation (Princeton, NJ), Autism Aid Onlus (Naples, Itay), and Fondazione Gaetano and Mafalda Luce (Milan, Itay).

1 American Psychiatric Association: American Psychiatric Association: Diagnostic and Statistical Manual of Mental Disorders 4th edition. Publisher, 1994.

2 Persico AM, Bourgeron T: Searching for ways out of the autism maze: genetic, epigenetic and environmental clues. Trends Neurosci 2006; 29: 349-358.

3 Klauck SM: Genetics of autism spectrum disorder. Eur J Hum Genet 2006; 14: 714-720.

4 Lintas C, Persico AM: Autistic phenotypes and genetic testing: state-of-the-art for the clinical geneticist. J Med Genet 2009; 46: 1-8.

5 Buxbaum JD: Multiple rare variants in the etiology of autism spectrum disorders. Dialogues Clin Neurosci 2009; 11: 35-43.

6 Cook EH, Leventhal BL: The serotonin system in autism. Curr Opin Pediatr 1996; 8: 348-354.

7 Abramson RK, Wright HH, Carpenter R et al: Elevated blood serotonin in autistic probands and their first-degree relatives. J Autism Dev Disord 1989; 19: 397-407.

8 Cook EH, Leventhal BL, Heller W, Metz J, Wainwright M, Freedman DX: Autistic children and their first-degree relatives: relationships between serotonin and norepinephrine levels and intelligence. J Neuropsychiatry Clin Neurosci 1990; 2: 268-274.

9 Leventhal BL, Cook Jr EH, Morford M, Ravitz A, Freedman DX: Relationships of whole blood serotonin and plasma norepinephrine within families. J Autism Dev Disord 1990; 20: 499-511.

10 Weiss LA, Veenstra-Vanderweele J, Newman DL et al: Genome-wide association study identifies ITGB3 as a QTL for whole blood serotonin. Eur J Hum Genet 2004; 12: 949-954.

11 Weiss LA, Abney M, Cook Jr EH, Ober C: Sex-specific genetic architecture of whole blood serotonin levels. Am J Hum Genet 2005; 76: 33-41.

12 Stone JL, Merriman B, Cantor RM et al: Evidence for sex-specific risk alleles in autism spectrum disorder. Am J Hum Genet 2004; 75: 1117-1123.

13 Cantor RM, Kono N, Duvall JA et al: Replication of autism linkage: fine-mapping peak at 17q21. Am J Hum Genet 2005; 76: 1050-1056.

14 Weiss LA, Ober C, Cook Jr EH: ITGB3 shows genetic and expression interaction with SLC6A4. Hum Genet 2006; 120: 93-100.

15 Coutinho AM, Sousa I, Martins M et al: Evidence for epistasis between SLC6A4 and ITGB3 in autism etiology and in the determination of platelet serotonin levels. Hum Genet 2007; 121: 243-256.

16 Weiss LA, Kosova G, Delahanty RJ et al: Variation in ITGB3 is associated with wholeblood serotonin level and autism susceptibility. Eur J Hum Genet 2006; 14: 923-931.

17 Mei H, Cuccaro ML, Martin ER: Multifactor dimensionality reduction-phenomics: a novel method to capture genetic heterogeneity with use of phenotypic variables. Am J Hum Genet 2007; 81: 1251-1261.

$18 \mathrm{Ma} \mathrm{DQ}$, Rabionet R, Konidari I et al: Association and gene-gene interaction of SLC6A4 and ITGB3 in autism. Am J Med Genet B Neuropsychiatr Genet 2009; 153: 477-483.

19 Carneiro AM, Cook EH, Murphy DL, Blakely RD: Interactions between integrin alphallbbeta3 and the serotonin transporter regulate serotonin transport and platelet aggregation in mice and humans. J Clin Invest 2008; 118: 1544-1552. 
20 Weiss LA, Abney M, Parry R, Scanu AM, Cook Jr EH, Ober C: Variation in ITGB3 has sex-specific associations with plasma lipoprotein(a) and whole blood serotonin levels in a population-based sample. Hum Genet 2005; 117: 81-87.

21 Conciatori M, Stodgell CJ, Hyman SL et al: Morphogenetic effect of the HOXA1 A218G polymorphism on head circumference in patients with autism. Biol Psychiatry 2004; 55: 413-419.

22 Lord C, Rutter M, DiLavore PC, Risi S: ADOS, Autism Diagnostic Observation Schedule. Western Psychological Services: Los Angeles, 2002 (Italian version ed. by Tancredi R, Saccani M, Persico AM, Parrini B, Igliozzi R and Faggioli R. Organizzazioni Speciali: Florence, 2005)

23 Rutter M, Le Couter A, Lord C: ADI-R, Autism Diagnostic Interview - Revised. Western Psychological Services: Los Angeles, 2003 (Italian version ed. by Faggioli R, Saccani M, Persico AM, Tancredi R, Parrini B and Igliozzi R. Organizzazioni Speciali: Florence, 2005).

24 Lahiri DK, Nurnberger Jr JI: A rapid non-enzymatic method for the preparation of HMW DNA from blood for RFLP studies. Nucleic Acids Res 1991; 19: 5444.

25 Barrett JC, Fry B, Maller J, Daly MJ: Haploview: analysis and visualization of LD and haplotype maps. Bioinformatics 2005; 21: 263-265.

26 Persico AM, Militerni R, Bravaccio $\mathrm{C}$ et al: Lack of association between serotonin transporter gene promoter variants and autistic disorder in two ethnically distinct samples. Am J Med Genet 2000; 96: 123-127.

27 Piven J, Tsai GC, Nehme E, Coyle JT, Chase GA, Folstein SE: Platelet serotonin, a possible marker for familial autism. J Autism Dev Disord 1991; 21: 51-59.

28 Reichelt WH, Knivsberg AM, Nodland M, Stensrud M, Reichelt KL: Urinary peptide levels and patterns in autistic children from seven countries, and the effect of dietary intervention after 4 years. Dev Brain Dysfunct 1997; 10: 44-55.

29 O'Connell JR, Weeks DE: PedCheck: a program for identification of genotype incompatibilities in linkage analysis. Am J Hum Genet 1998; 63: 259-266.

30 Gabriel SB, Schaffner SF, Nguyen $\mathrm{H}$ et al: The structure of haplotype blocks in the human genome. Science 2002; 296: 2225-2229.

31 Wang N, Akey JM, Zhang K, Chakraborty R, Jin L: Distribution of recombination crossovers and the origin of haplotype blocks: the interplay of population history, recombination, and mutation. Am J Hum Genet 2002; 71: 1227-1234.

32 Horvath S, Xu X, Lake SL, Silverman EK, Weiss ST, Laird NM: Family-based tests for associating haplotypes with general phenotype data: application to asthma genetics. Genet Epidemiol 2004; 26: 61-69.
33 Dudbridge F: Pedigree disequilibrium tests for multilocus haplotypes. Genet Epidemiol 2003; 25: 115-121.

34 Cordell HJ, Barratt BJ, Clayton DG: Case/pseudocontrol analysis in genetic association studies: a unified framework for detection of genotype and haplotype associations, gene-gene and gene-environment interactions, and parent-of-origin effects. Genet Epidemiol 2004; 26: 167-185.

35 Sacco R, Militerni R, Frolli A et al: Clinical, morphological, and biochemical correlates of head circumference in autism. Biol Psychiatry 2007; 62: 1038-1047.

36 Nyholt NR: A simple correction for multiple testing for single-nucleotide polymorphisms in linkage disequilibrium with each other. Am J Hum Genet 2004; 74: 765-769.

37 Weiss LA, Lester LA, Gern JE et al: Variation in ITGB3 is associated with asthma and sensitization to mold allergen in four populations. Am J Respir Crit Care Med 2005; 172: 67-73.

38 Thompson EE, Pan L, Ostrovnaya I et al: Integrin beta 3 genotype influences asthma and allergy phenotypes in the first 6 years of life. J Allergy Clin Immunol 2007; 119: 1423-1429.

39 Neale BM: Linkage disequilibrium and tagging; in Neale BM, Ferreira MAR, Medland SE, Posthuma D (eds): Statistical Genetics: Gene Mapping Through Linkage and Association. Taylor and Francis, Inc: London, 2007, pp 451-465.

40 Dreyfus M, Kaplan C, Verdy E et al: Frequency of immune thrombocytopenia in newborns: a prospective study. Immune Thrombocytopenia Working Group. Blood 1997; 89: 4402-4406.

41 Ruzzi L, Ciarafoni I, Silvestri L, Semeraro ML, Abeni D: Association of PLA2 polymorphism of the ITGB3 gene with early fetal loss. Fertil Steril 2005; 83: 511-512.

42 O'Connell PJ, Wang X, Leon-Ponte M, Griffiths C, Pingle SC, Ahern GP: A novel form of immune signaling revealed by transmission of the inflammatory mediator serotonin between dendritic cells and T cells. Blood 2006; 107: 1010-1017.

43 Kundermann B, Hemmeter-Spernal J, Strate $\mathrm{P}$ et al: Pain sensitivity in major depression and its relationship to central serotoninergic function as reflected by the neuroendocrine response to clomipramine. J Psychiatr Res 2009; 43: 1253-1261.

44 Husebekk A, Killie MK, Kjeldsen-Kragh J, Skogen B: Is it time to implement HPA-1 screening in pregnancy? Curr Opin Hematol 2009; 16: 497-502.

Supplementary Information accompanies the paper on European Journal of Human Genetics website (http://www.nature.com/ejhg) 